\title{
Heterogeneity of clinical features and mutation analysis of NTRKI in Han Chinese patients with congenital insensitivity to pain with anhidrosis
}

This article was published in the following Dove Medical Press journal: Journal of Pain Research

\author{
Ningbo Li' \\ Shanna Guo' \\ Qingli Wang ${ }^{2}$ \\ Guangyou Duan ${ }^{3}$ \\ Jiaoli Sun' \\ Yi Liu' \\ Jin Zhang' \\ Cong Wang' \\ Changmao Zhu' \\ jingyu Liu ${ }^{4}$ \\ Xianwei Zhang' \\ 'Department of Anesthesiology, \\ Tongji Hospital, Tongji Medical \\ College, Huazhong University \\ of Science and Technology, \\ Wuhan, China; ${ }^{2}$ Department of \\ Anesthesiology, Wuhan General \\ Hospital of Guangzhou Military, \\ Wuhan, China; ${ }^{3}$ Department of \\ Anesthesiology, Xinqiao Hospital, \\ Third Military Medical University, \\ Chongqing, China; ${ }^{4}$ Key Laboratory \\ of Molecular Biophysics of Ministry \\ of Education, College of Life Science \\ and Technology, Center for Human \\ Genome Research, Huazhong \\ University of Science and Technology, \\ Wuhan, China
}

Correspondence: Xianwei Zhang Department of Anesthesiology, Tongji Hospital, Tongji Medical College, Huazhong University of Science and Technology, No 1095 Jie-Fang Road, Wuhan 430030, China

$\mathrm{Tel}+86 \quad$ I30 37I5 4560

Fax +862783662853

Email ourpain@163.com
Purpose: Congenital insensitivity to pain with anhidrosis (CIPA) is a rare inherited disorder whose core clinical features consist of no response to noxious stimuli and inability to sweat under any conditions. Our goal was to characterize the details of phenotypic and genotypic features in Chinese CIPA patients.

Patients and methods: Personal data and clinical information were investigated by interview and physical examination. DNA was extracted from blood samples of patients and their available familial members and subjected to genetic analysis.

Results: A total of 41 Han Chinese CIPA patients from 35 unrelated families were recruited. The distribution of patients was mainly in the central and southern regions of China, with a male to female ratio of $3: 1$ and a mortality rate of $7.3 \%$. Heterogeneity of clinical features, including pain insensitivity, temperature sensation, and complications, were cataloged. Interestingly, some patients had "visceral pain" sensation, and there was a significant difference in temperature perception and thermal pain between individuals. The incidence of bone and joint fractures was $49 \%$. The characteristics of 19 mutations of NTRK1 in 41 patients, with five novel mutations, were identified. More than $63 \%$ of patients had the splice mutation, c.851-33 T>A, which strongly suggests that it may be a common pathogenic site in Han Chinese patients.

Conclusion: Current findings expand our knowledge about the spectrum of phenotypic features and the racial characteristics of NTRK1 mutations of CIPA patients in the Han Chinese population. Keywords: congenital insensitivity to pain with anhidrosis, HASN IV, phenotype, NTRK1, mutation, Chinese

\section{Introduction}

Congenital insensitivity to pain with anhidrosis (CIPA; OMIM 256800), also known as hereditary sensory and autonomic neuropathy type IV (HSAN IV), was first reported in 1963; it is a rare autosomal recessive genetic disorder that is caused by the failure of nociceptive and sympathetic neuron development. ${ }^{1,2}$ In 1996, Indo et al $^{3}$ first identified neurotrophic tyrosine kinase receptor type 1 (NTRK1) mutations in three unrelated CIPA patients. The gene NTRK1 (MIM\#191315) located on chromosome 1q21-q22 includes 17 exons and yields a 796-residue protein (tropomyosin-related kinase A [TrkA]). TrkA protein specifically binds to extracellular nerve growth factor (NGF), stimulates homodimer formation, and activates its tyrosine kinase activity, resulting in phosphorylation of specific tyrosine residues in the intracellular domain. The lack of pain sensation and the presence of anhidrosis in CIPA are caused by the absence of NGF-dependent primary afferent neurons with unmyelinated C-fibers and sympathetic 
postganglionic neurons, respectively. ${ }^{4}$ To date, more than 105 NTRK 1 mutations have been reported in CIPA patients. ${ }^{5}$

The main clinical features of this condition are congenital, and they include complete insensitivity to both superficial and deep painful stimuli, absence of sweating, and recurrent episodes of unexplained fever. Moreover, these patients exhibit self-mutilating behaviors such as biting their fingers, lips, and tips of the tongue, and they frequently present with varying degrees of trauma resulting from falls, burns, and bone and joint fractures. Key features of this condition include a deficit in temperature perception and variable mental retardation. In addition to the abovementioned common symptoms, each patient presents with unique clinical features, such as thin hair, eczema, glaucoma, and so on. ${ }^{6}$ However, NTRK1-NGF signaling, which is an important signaling pathway in vivo, participates in many physiological processes; thus, disruption of these processes results in many of the pathological features found in CIPA. Many of the clinical symptoms of CIPA are not fully recognized.

Although the disease has been reported all over the world, CIPA is currently considered to be rare and sporadic in most populations. Israel and Japan have reported dozens of cases, and others have been reported in Pakistan, Korea, and Malaysia. Epidemiological surveys conducted by Japanese scholars estimated that there were approximately 130-210 CIPA patients in Japan in 2009. ${ }^{7}$ Studies from Japan and Israel reported common pathogenic mutations. In 2000 and 2001, Japan and Israel described the clinical phenotype and genotype of CIPA patients with various ethnic characteristics. ${ }^{8-10}$ Three variants - p.Phe284TrpfsTer36, p.Arg554GlyfsTer104, and p.Asp674Tyr - accounted for roughly $70 \%$ of pathogenic NTRK1 alleles in the Japanese population. ${ }^{11}$ One variant, p.Pro621SerfsTer12, accounted for $89 \%$ of pathogenic NTRK1 alleles among Israeli Bedouins. ${ }^{8,10}$ However, details of the clinical features and mutation characteristics in Chinese CIPA patients remain unclear.

Since the first reported cases of a Han-Chinese CIPA patient in 1983 and the NTRK1 gene mutation in Taiwan in 2010, ${ }^{12}$ Chinese CIPA patients have been reported for decades, but the numbers are extremely rare. ${ }^{13-15}$ Articles in the literature are limited to descriptions of disease symptoms and pathogenic mutations in the form of case reports. Thus, additional research is needed to expand our knowledge on the spectrum of phenotypic and genotypic characteristics found in Chinese patients with CIPA, to better facilitate clinical diagnosis and genetic counseling. Chinese patients with CIPA could exhibit their own clinical phenotype and genotype characteristics.
Therefore, in this study, we examined the unique clinical symptoms and genotypic features of 41 Chinese patients with CIPA from 35 unrelated families.

\section{Patients and methods}

This study was approved by the institutional ethics committee at Tongji Hospital, Tongji Medical College, Huazhong University of Science and Technology (20130501). Written informed consent was obtained from all participants and parents or legal guardians for participants younger than 18 years, and this study was conducted in accordance with the Declaration of Helsinki.

\section{Subjects and phenotype}

The current study included 41 cases of patients with CIPA, identified and collected between January 2013 and February 2018, by means of hospitals records, social networking platforms, and news reports. Medical history records and questionnaires were used to collect information regarding the discovery process, clinical experience, peripheral and central nervous system, skeletal system, immune system, developmental history, and other specific symptoms and signs. The quantitative sensory testing, including mechanical pain and thermal pain, sweat test, nerve biopsy, etc., were performed in certain conditions. ${ }^{16}$ The diagnosis was made based on clinical findings, the results of physiological testing, and sometimes nerve biopsy and/or gene analyses.

\section{Genetic analysis}

Peripheral blood was collected from all patients and available family members after informed consent was received. Genomic DNA was extracted from blood samples using the QIAamp DNA Blood Mini Kit (Qiagen NV, Venlo, the Netherlands) according to the manufacturer's instructions. Patients were tested on a targeted next-generation sequencing (NGS) gene panel, which contains 57 genes associated with known causes of inherited peripheral neuropathy, provided by the Beijing Genomics Institute. Library preparation and sequencing were performed using the Illumina MiSeq platform (Illumina Inc., San Diego, CA, USA) according to the manufacturer's standard protocol.

The resulting sequences were analyzed using sequencing analysis (version 5.1.1) and compared with the current reference sequence for NTRK1 in the National Center for Biotechnology Information (NCBI) database (NM_002529.3), corresponding to the amino sequence NP_002520.2. Sequencing reactions and alignments to the reference human transcriptome were repeated twice. For novel variants, 
molecular consequences were inferred by variation reporter based on RefSeq transcripts.

\section{Results}

\section{Demographic data}

A total of 44 patients with suspected CIPA were identified. Three cases of purely non-hyperhidrosis patients (probably ectodermal dysplasia) were excluded from the study through the analysis of clinical phenotypes collected from the medical history and questionnaires. In this study, clinical features and genetic analyses were performed on the remaining 41 patients.

All 41 cases of CIPA occurred sporadically in 35 unrelated non-consanguineous marriage families, of which three patients (F-2, F-12, and F-35-1) had passed away. Five patients (F-9, F-5, F-16, F-23, and F-25) have been reported in our previous studies. ${ }^{6,17}$ The patients were mainly distributed in the central and southern regions of China, and all of them were Han Chinese. A detailed geographical distribution map is shown in Figure 1A. The age of the 41 patients ranged from 2 to 19 years ( $5.65 \pm 4.64$ years), with $70 \%$ of the population aged 2-6 years (Figure 1B). None of them had married or given childbirth. The study group comprised 30 males and 11 females, with a gender ratio of 3:1 (Figure 1C). The mortality rate in this study group was $7.3 \%$.

\section{Heterogeneity of physical characteristics}

The medical history and clinical information forms of 35 patients (three patients died and three patients were lost to
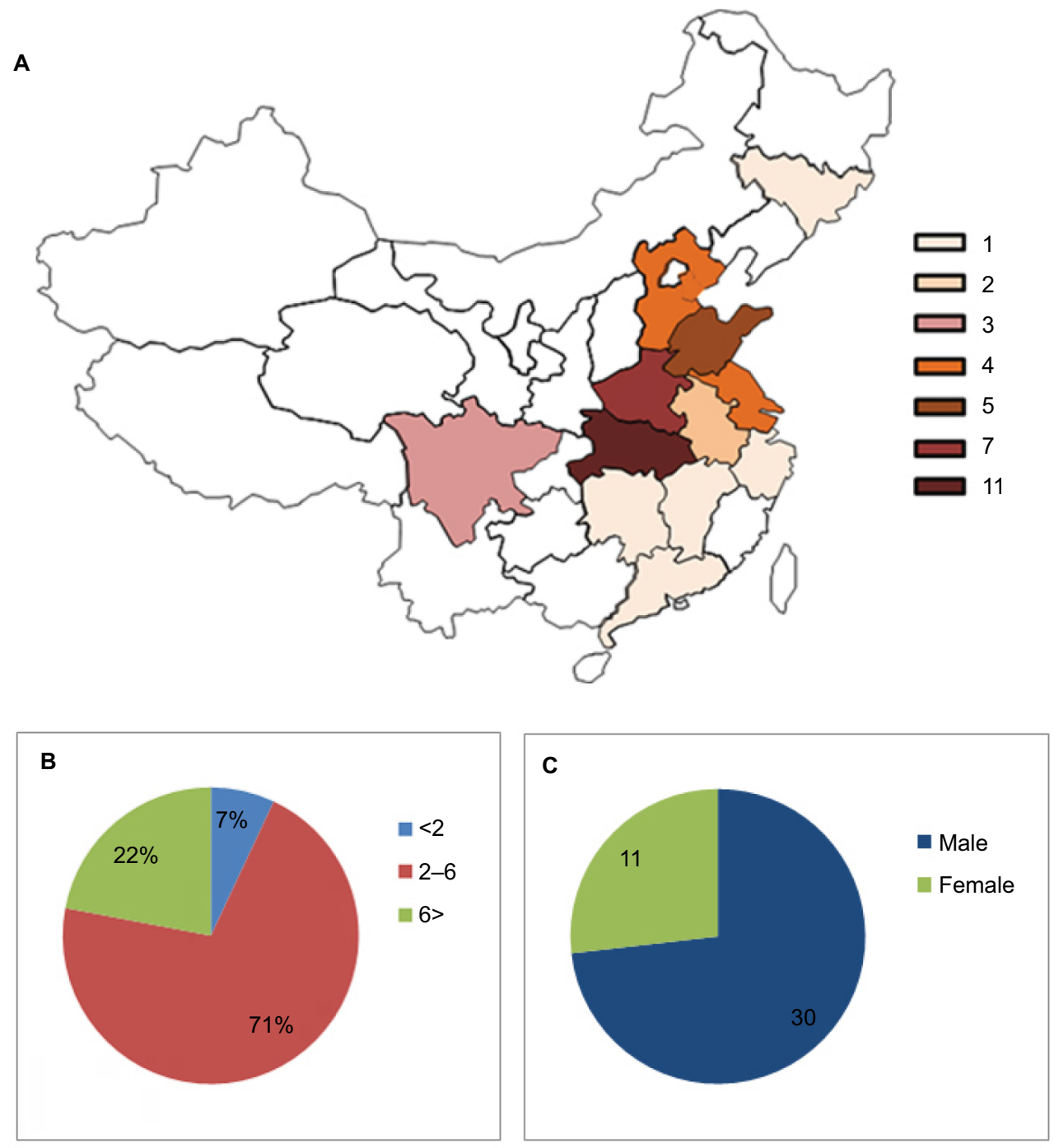

Figure I Epidemiological data of CIPA patients in this study.

Notes: (A) Geographical distribution map of patients with congenital insensitivity to pain and anhidrosis in China. (B) Age profile of the patients. The data are expressed as a percentage. (C) Gender profile of the patients. The gender distribution of the patients was 30 for male and II for female. Abbreviation: CIPA, congenital insensitivity to pain with anhidrosis. 
follow-up) were collected. All patients presented with typical clinical manifestations of the disease - insensitivity to noxious stimuli, anhidrosis, and recurring high fevers. Almost all the patients had suffered from bruising, repeated fractures, and bodily injury from biting their fingers, lips, and tips of the tongue during the teething stage (Figure 2). The detailed manifestations observed in the patients are shown in Table 1.

Based on the data collected, the patients showed complete insensitivity to superficial painful stimuli. However, of the 35 patients, 12 and eight patients reported "stomachache" and "cephalofacial pain" during their growth period, respectively (Table 1). Pain sensation response to physical attack could be elicited in areas around the skin of the eyelids and over the bridge of the nose. Interestingly, a pair of sisters (F-16-1, F-16-2) who were diagnosed with CIPA "recovered" their pain perception after 10 years of age. The patients' temperature perception is an interesting phenomenon. According to parent reports, the temperature perception of a few patients was normal, and most patients did not respond to cold stimulation (data were not shown). We conducted detailed temperature perception tests on five patients and found that the test results were very different (Table 2). On meticulous observation, one could elicit several responses to cold and heat stimulation in CIPA patients. Patient F-3 could perceive cold and warm sensations, and there was escape response to a $48^{\circ} \mathrm{C}$ heat stimulus. The other four patients (F-4, F-10, F-281, and F-28-2) showed impaired temperature perception, but perception of warm stimuli on the face was basically normal. In general, they could distinguish between warm and cold stimuli on their faces during testing but failed to do so with the dorsum of their hands and feet. Additionally, thermal pain sensation and cold pain sensation were absent.

Most patients had episodes of unexplained fever; seven of the 35 patients had a history convulsions or coma due to high fever, and one patient was misdiagnosed as having an infection due to postnatal hyperthermia. Additionally, improper dressing had led to montmorillonite hypoxia syndrome and respiratory failure. The main cause of death in CIPA patients in the early period is high fever, but only three (F2-1, F12, and F35-1) deaths due to high fever were reported in the medical history we collected. Heat, chemical, and emotional sweating was completely absent. However, slight moisture in the intertriginous areas of the skin of the apex nasi, axillae, and groin could be detected, likely reflecting delayed evaporation of insensible water. Almost all patients were heat intolerant.

Repeated multiple fractures, hip dislocation, and osteomyelitis primarily occurred in the long bones and calcaneus of the lower extremities (Figure 2). The vast majority of patients did not experience the intense external impacts that resulted in fractures. These fractures frequently and inadvertently occurred when the patients began to walk, and they occurred in multiple locations in subsequent years. Additionally, these patients often suffered from unexplained non-infectious knee and ankle swelling, causing limb deformities and lameness (Figure 2D-F). The incidence of fractures in our patients was $49 \%$. Moreover, one interesting phenomenon is that the patients' lower limbs have a poor sense of weakness, which puts them at a greater risk for falls than their peers; however, their electromyography and motor nerve conduction test results are in the normal range. Based on our limited knowledge of the pathophysiology of this disease, the cause of this phenomenon is not fully understood, and it deserves further exploration.

Repeated infections such as osteomyelitis, pneumonia, and epidermal ulceration are common symptoms. Additionally, patients exhibit slow wound healing, which may be due to a lack of pain awareness and protection, but the possibility of reduced immune and self-healing capacity is not ruled out.

Patients with CIPA showed different degrees of mental retardation, especially with regard to language and adaptability. However, four patients exhibited normal mental development with no apparent delays. According to the results of our questionnaire and interview, most of the patients are hyperactive, irritable, and emotionally changeable, while a few appear to be hypoactive. In addition, these patients have compulsive behaviors that they are unable to control. Figure 2B shows the palm of a 10-year-old normal-intelligence boy (F-4) who cannot restrain himself from licking his fingers, which has resulted in defects in the ends of his ten fingers. In severe cases, tooth extraction is required to prevent these situations from deteriorating. Three patients in this study had a history of repeated finger biting, resulting in infection and truncation.

Finally, it is imperative that pediatricians be aware of the fact that the majority of patients with this condition are frequently diagnosed with severe neonatal pneumonia due to repeated high fevers after birth, until later in life when anhidrosis is detected. In addition, these patients are diagnosed with ectodermal dysplasia and diabetes insipidus.

\section{Mutations in NTRKI in CIPA patients}

In the current study, mutations in the NTRK1 gene were screened in 41 patients from 35 unrelated CIPA families using different detection methods. Through the two sequence 
A

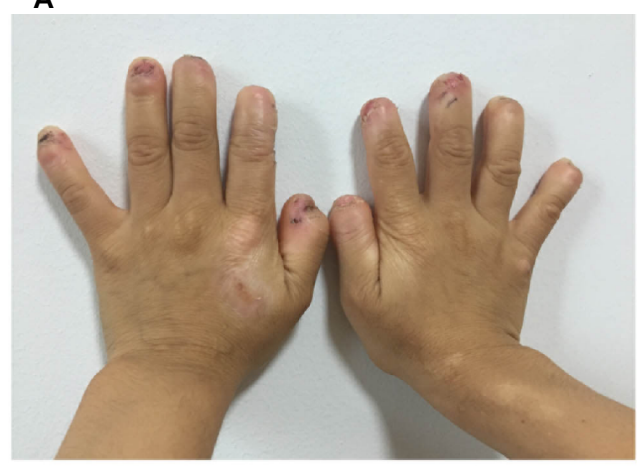

D

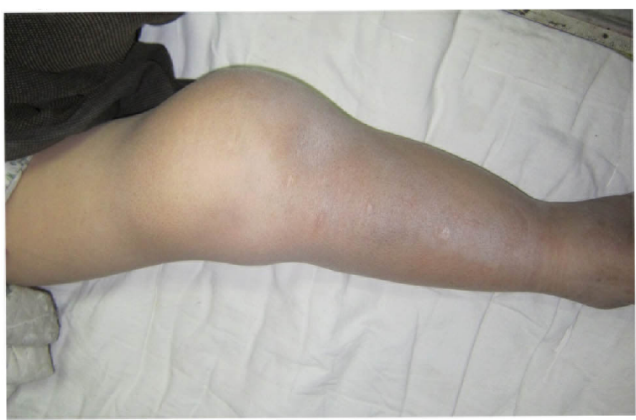

G

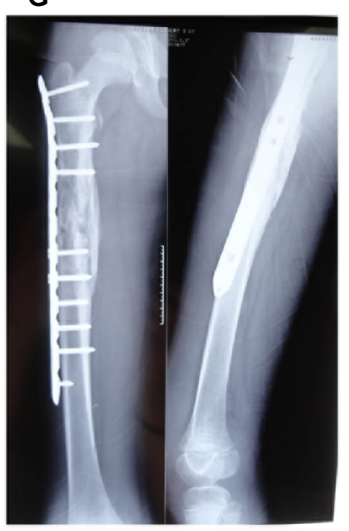

K

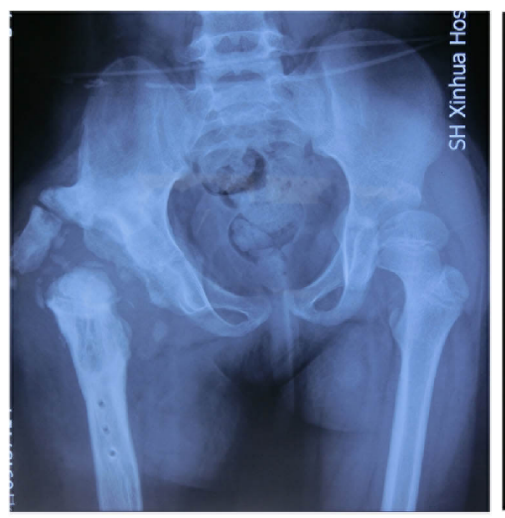

L
B

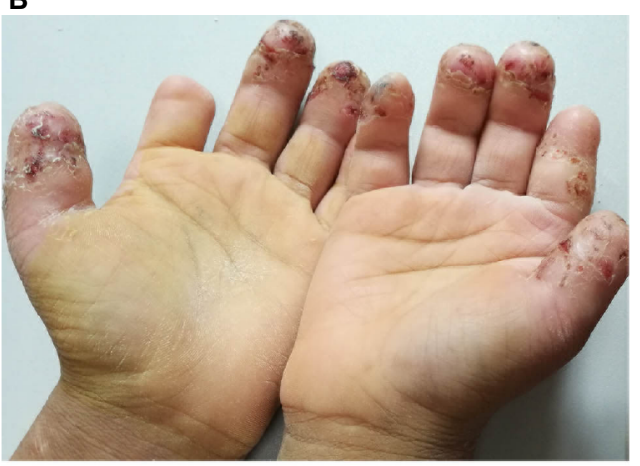

E

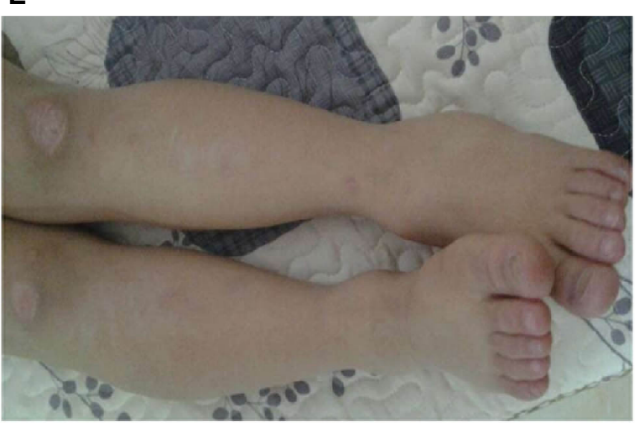

C

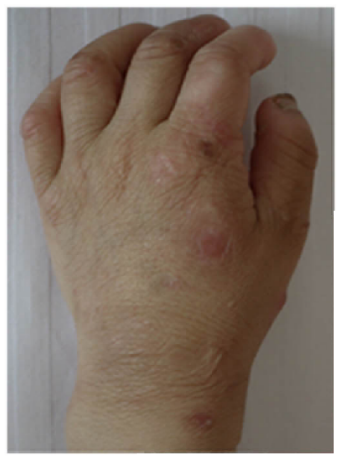

F

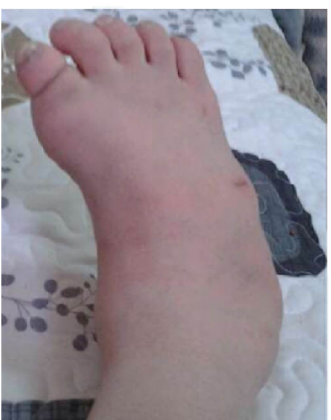

I
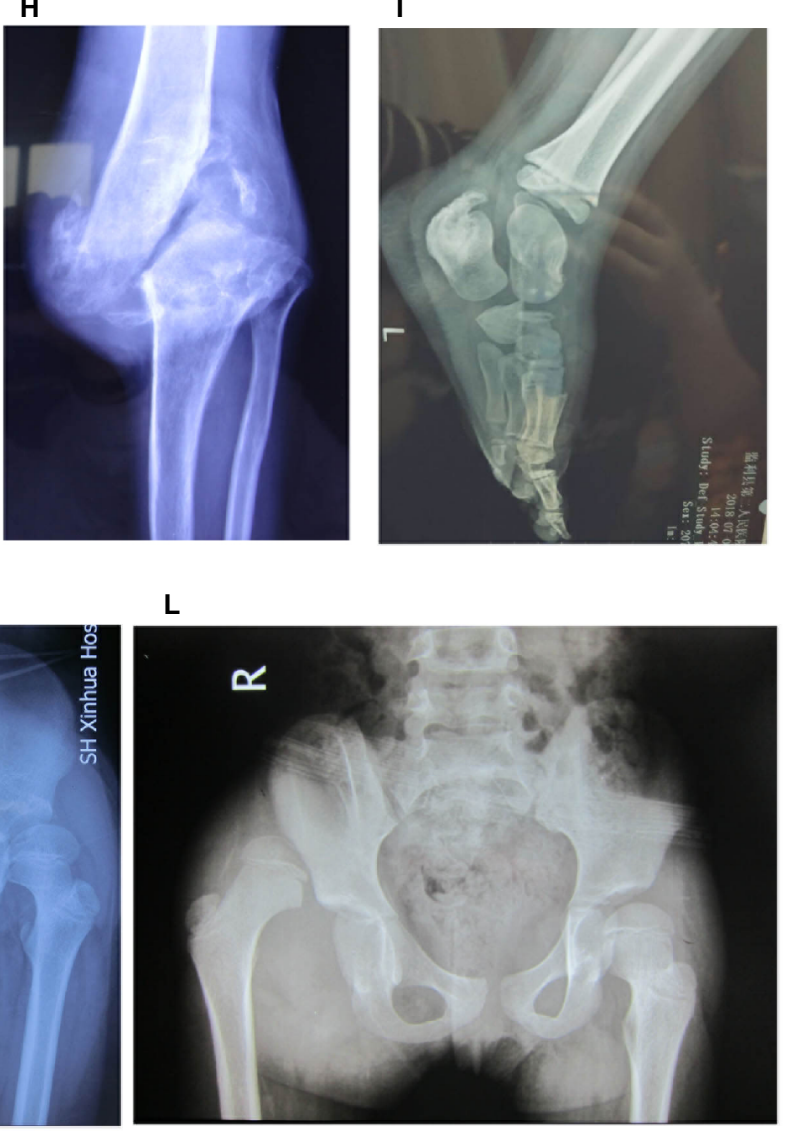

J

M

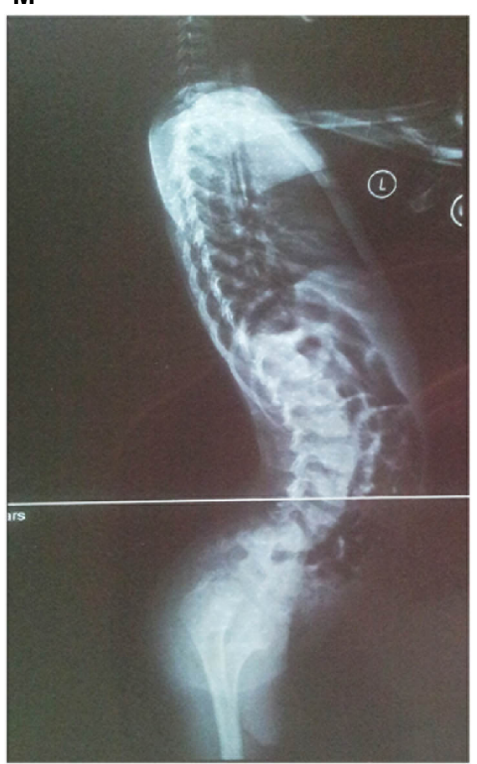

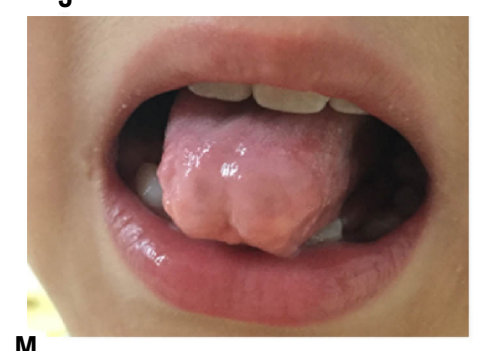

Figure 2 Pictures depicting common clinical features of patients.

Notes: (A) Hands showing damage to the fingertips after repeated finger biting (F-9). (B) Repeated cracking and infection of the fingers resulting from bruising and delayed healing (F-4). (C) The skin of the hand is thickened and dry (F-24-2). (D-F) Knee and ankle joints are swollen and deformed (F-8, F-24-I, F-24-2). (G-I) Radiographs showing repeated multiple fractures (F-I4, F-I8, F-3I). (J) Truncation of the tongue resulting from tongue biting (F-3). (K-L) Radiographs showing hip dislocation and fracture (F-I6-2, $\mathrm{F}-\mathrm{I0}$ ). (M) Radiograph showing the pathological process of the lumbar vertebra bending inward (F-25). 
Table I Mutations and clinical manifestations of the Han Chinese patients with congenital insensitivity to pain and anhidrosis

\begin{tabular}{|c|c|c|c|c|c|c|c|}
\hline \multirow{2}{*}{$\begin{array}{l}\text { Family } \\
\text { no. }\end{array}$} & \multirow[t]{2}{*}{ Sex } & \multirow{2}{*}{$\begin{array}{l}\text { Age } \\
\text { (years) }\end{array}$} & \multirow{2}{*}{$\begin{array}{l}\text { Mutation } \\
\text { origin }\end{array}$} & \multirow[t]{2}{*}{ Region } & \multirow[t]{2}{*}{ Nucleotide change } & \multicolumn{2}{|c|}{ Pain sensation } \\
\hline & & & & & & Absent & $\begin{array}{l}\text { Cephalofacial } \\
\text { pain }\end{array}$ \\
\hline 1 & M & 3 & $\begin{array}{l}\text { Het-F } \\
M\end{array}$ & $\begin{array}{l}\text { Intron } 2 \\
\text { Exon } 14\end{array}$ & $\begin{array}{l}\text { c. } 287+2 \text { dupT } \\
\text { c. } 1775 \text { T > G (p.M592R) }\end{array}$ & + & + \\
\hline $2-I^{a}$ & M & $8 \mathrm{~m}$ & Hom F/M & Exon 5 & c.429-374_c.7I7+485del I403 (p.VI44Nfs'8) & + & UN \\
\hline $2-2$ & $M$ & 5 & Hom F/M & Exon 5 & c.429-374_c.7I7+485del I403 (p.VI44Nfs'8) & + & + \\
\hline 3 & $\mathrm{~F}$ & 5 & Het-M & Exon 5 & c.446_447insTCTG (p.PI49fs) & + & + \\
\hline 4 & M & 10 & Het-F & Exon 16 & c.2074C>T (p.R692C) & + & - \\
\hline 5 & M & 8 & $\begin{array}{l}\text { Het-F } \\
M\end{array}$ & $\begin{array}{l}\text { Exon } 16 \\
\text { Exon } 17\end{array}$ & $\begin{array}{l}\text { c.2074C >T (p.R692C) } \\
\text { c.23IIIC>T (p.R77|C) }\end{array}$ & + & - \\
\hline 6 & $M$ & 2 & Het-F & Intron 7 & c. $85 \mathrm{I}-33 \mathrm{~T}>\mathrm{A}$ & + & - \\
\hline $7^{b}$ & $M$ & 3 & Het-M & Intron 2 & c.287+2dupT & + & UN \\
\hline 8 & $M$ & 11 & Het-M & Intron 7 & c.85I-33T $>A$ & + & - \\
\hline 9 & $M$ & 3 & $\begin{array}{l}\text { Het-F } \\
M\end{array}$ & $\begin{array}{l}\text { Intron } 7 \\
\text { Exon } 16\end{array}$ & $\begin{array}{l}\text { c.85I-33T>A } \\
\text { c.2197G >A (p.G7|4D) }\end{array}$ & + & - \\
\hline 10 & $\mathrm{~F}$ & 19 & \multicolumn{3}{|l|}{ UN } & + & - \\
\hline $1 \mathrm{I}^{\mathrm{b}}$ & $M$ & 3 & Het-F & Intron 7 & c. $85 \mathrm{I}-33 \mathrm{~T}>\mathrm{A}$ & + & - \\
\hline $12^{\mathrm{a}}$ & $\mathrm{F}$ & 5 & Het-F & Intron 7 & c. $85 \mathrm{I}-33 \mathrm{~T}>\mathrm{A}$ & + & UN \\
\hline 13 & $M$ & 5 & $\begin{array}{l}\text { Het-F } \\
M\end{array}$ & $\begin{array}{l}\text { Intron } 7 \\
\text { Exon } 13\end{array}$ & $\begin{array}{l}\text { c.85I-33T>A } \\
\left.\text { c. 1736delT (p.L579Rfs }{ }^{\mathrm{b}} 73\right)\end{array}$ & + & - \\
\hline 14 & $\mathrm{~F}$ & 9 & Het-M & Intron 7 & c. $85 \mathrm{I}-33 \mathrm{~T}>\mathrm{A}$ & + & - \\
\hline 15 & $M$ & 3 & $\begin{array}{l}\text { Het-F } \\
M\end{array}$ & $\begin{array}{l}\text { Intron } 7 \\
\text { Intron } 2 \\
\end{array}$ & $\begin{array}{l}\text { c.85I-33T >A } \\
\text { c. } 287+2 \text { dupT } \\
\end{array}$ & + & - \\
\hline $16-1$ & $\mathrm{~F}$ & 19 & Hom F/M & Intron 7 & c.85I-33T $>A$ & + & + \\
\hline $16-2$ & $\mathrm{~F}$ & 11 & Hom F/M & Intron 7 & c. $85 \mathrm{I}-33 \mathrm{~T}>\mathrm{A}$ & + & - \\
\hline 17 & $M$ & 4 & \multicolumn{3}{|l|}{ UN } & + & - \\
\hline 18 & $M$ & 19 & \multicolumn{3}{|l|}{ UN } & + & - \\
\hline $19^{b}$ & $M$ & 3 & $\begin{array}{l}\text { Het-F } \\
M\end{array}$ & $\begin{array}{l}\text { Exon } 17 \\
\text { Intron } 14\end{array}$ & $\begin{array}{l}\text { c. } 23 \mid 2 G>A(p . R 77 \mid H) \\
\text { c. } 1806-2 A>G\end{array}$ & + & - \\
\hline 20 & $M$ & 1.5 & \multicolumn{3}{|l|}{ UN } & + & + \\
\hline 21 & $M$ & 5 & \multicolumn{3}{|l|}{ UN } & + & - \\
\hline 22 & $M$ & $5 \mathrm{~m}$ & Het-M & Intron 7 & c. $85 \mathrm{I}-33 \mathrm{~T}>\mathrm{A}$ & + & - \\
\hline 23 & $M$ & 2 & $\begin{array}{l}\text { Het-F } \\
M\end{array}$ & $\begin{array}{l}\text { Exon } 8 \\
\text { Intron } 7 \\
\end{array}$ & $\begin{array}{l}\text { c.963delG (p.L322Sfs }{ }^{\mathrm{l}} \text { |48) } \\
\text { c.85I-33T>A }\end{array}$ & + & + \\
\hline $24-1$ & $M$ & 12 & Het-F & Intron 7 & c. $85 \mathrm{I}-33 \mathrm{~T}>\mathrm{A}$ & + & - \\
\hline $24-2$ & $M$ & 6 & Het-F & Intron 7 & c.85I-33T >A & + & - \\
\hline 25 & $M$ & 6 & $\begin{array}{l}\text { Het-F } \\
M\end{array}$ & $\begin{array}{l}\text { Exon } 14 \\
\text { Exon } 14\end{array}$ & $\begin{array}{l}\text { c. } 1802 \mathrm{~T}>\mathrm{G}(\mathrm{p} . \mathrm{L} 60 \mathrm{IR}) \\
\text { c. } 1804 \mathrm{C}>\mathrm{T} \text { (p.R602fs) }\end{array}$ & + & - \\
\hline 26 & $F$ & 2 & $\begin{array}{l}\text { Het-F } \\
M\end{array}$ & $\begin{array}{l}\text { Exon } 13 \\
\text { Intron } 7\end{array}$ & $\begin{array}{l}\text { c.I7II_I72Idel (p.G57IRfs } 10) \\
\text { c.85I-33T>A }\end{array}$ & + & - \\
\hline 27 & $\mathrm{~F}$ & 5 & $\begin{array}{l}\text { Het-F } \\
M\end{array}$ & $\begin{array}{l}\text { Intron } 7 \\
\text { Exon } 6\end{array}$ & $\begin{array}{l}\text { c.85I-33T }>A \\
\text { c.632T }>A(p . V 2 I \mid I E)\end{array}$ & + & - \\
\hline $28-1$ & $M$ & 4 & $\begin{array}{l}\text { Het-F } \\
M\end{array}$ & $\begin{array}{l}\text { Exon } 6 \\
\text { Exon II }\end{array}$ & $\begin{array}{l}\text { c.632T>A (p.V2IIE) } \\
\text { c. 1253_I254delTC (p.S419Gfs } 80)\end{array}$ & + & - \\
\hline $28-2$ & $M$ & 4 & $\begin{array}{l}\text { Het-F } \\
M\end{array}$ & $\begin{array}{l}\text { Exon } 6 \\
\text { Exon II }\end{array}$ & $\begin{array}{l}\text { c.632T>A (p.V2IIE) } \\
\text { c.1253_I254delTC (p.S4I9Gfsbo) }\end{array}$ & + & - \\
\hline 29 & $M$ & 2 & $\begin{array}{l}\text { Het-F } \\
M\end{array}$ & $\begin{array}{l}\text { Intron } 7 \\
\text { Exon } 14\end{array}$ & $\begin{array}{l}\text { c.85I-33T>A } \\
\text { c. } 1805 \mathrm{G}>\mathrm{A}(\mathrm{p} . \mathrm{R} 602 \mathrm{Q})\end{array}$ & + & + \\
\hline $30-1$ & $M$ & 2 & $\begin{array}{l}\text { Het-F } \\
M\end{array}$ & $\begin{array}{l}\text { Intron } 7 \\
\text { Exon } 16\end{array}$ & $\begin{array}{l}\text { c.85I-33T }>A \\
\text { c.2066C }>T \text { (p.P689L) }\end{array}$ & + & - \\
\hline $30-2$ & $M$ & 6 & $\begin{array}{l}\text { Het-F } \\
M\end{array}$ & $\begin{array}{l}\text { Intron } 7 \\
\text { Exon } 16\end{array}$ & $\begin{array}{l}\text { c.85I-33T >A } \\
\text { c.2066C >T (p.P689L) }\end{array}$ & + & - \\
\hline 31 & $M$ & 5 & $\begin{array}{l}\text { Het-F } \\
M\end{array}$ & $\begin{array}{l}\text { Exon } 5 \\
\text { Intron } 2 \\
\end{array}$ & $\begin{array}{l}\text { c.446_447insTCTG (p.PI49fs) } \\
\text { c.287+2dupT }\end{array}$ & + & - \\
\hline
\end{tabular}




\begin{tabular}{|c|c|c|c|c|c|c|c|c|}
\hline $\begin{array}{l}\text { Stomach } \\
\text { ache }\end{array}$ & No sweat & $\begin{array}{l}\text { Recurrent } \\
\text { fever }\end{array}$ & $\begin{array}{l}\text { Self- } \\
\text { mutilation }\end{array}$ & $\begin{array}{l}\text { Slow } \\
\text { wound } \\
\text { healing }\end{array}$ & $\begin{array}{l}\text { Bone } \\
\text { fractures }\end{array}$ & $\begin{array}{l}\text { Fall } \\
\text { dawn }\end{array}$ & $\begin{array}{l}\text { Severe } \\
\text { infection }\end{array}$ & $\begin{array}{l}\text { Mental } \\
\text { retardation }\end{array}$ \\
\hline+ & + & + & + & + & - & + & - & + \\
\hline UN & + & + & UN & UN & - & UN & UN & UN \\
\hline+ & + & + & + & + & + & + & - & + \\
\hline+ & + & ++ & + & - & - & - & + & + \\
\hline+ & + & + & + & + & + & - & ++ & - \\
\hline- & + & +++ & + & + & + & + & ++ & + \\
\hline- & + & + & + & - & - & + & + & + \\
\hline UN & + & + & + & UN & UN & UN & UN & UN \\
\hline- & + & ++ & + & + & + & + & + & + \\
\hline- & + & ++ & + & - & + & + & - & + \\
\hline- & + & ++ & + & + & ++ & + & + & + \\
\hline- & + & + & UN & UN & UN & UN & UN & UN \\
\hline UN & + & + & UN & UN & - & UN & UN & UN \\
\hline- & + & + & + & - & - & - & + & + \\
\hline+ & + & + & + & + & ++ & - & + & + \\
\hline- & + & + & + & - & - & + & - & + \\
\hline+ & + & + & + & - & + & - & - & - \\
\hline+ & + & + & + & - & ++ & + & + & - \\
\hline- & + & + & + & + & ++ & + & + & + \\
\hline- & + & + & + & - & + & - & + & + \\
\hline- & + & + & UN & UN & + & UN & UN & UN \\
\hline+ & + & + & + & + & + & + & - & + \\
\hline- & + & + & + & - & ++ & + & - & + \\
\hline- & + & + & + & + & - & UN & - & UN \\
\hline+ & + & + & + & - & - & + & - & + \\
\hline- & + & + & + & + & + & + & - & + \\
\hline- & + & + & + & + & ++ & - & - & + \\
\hline- & + & ++ & + & - & ++ & - & - & + \\
\hline- & + & + & + & - & - & + & - & + \\
\hline- & + & + & + & - & + & + & - & + \\
\hline- & + & ++ & + & + & - & - & - & + \\
\hline+ & + & ++ & + & + & - & - & - & + \\
\hline- & + & + & + & - & - & + & - & + \\
\hline- & + & + & + & + & - & + & + & + \\
\hline- & + & + & + & + & - & + & + & + \\
\hline- & + & + & + & - & ++ & + & - & - \\
\hline
\end{tabular}


Table I (Continued)

\begin{tabular}{|l|l|l|l|l|l|l|l|}
\hline $\begin{array}{l}\text { Family } \\
\text { no. }\end{array}$ & Sex & $\begin{array}{l}\text { Age } \\
\text { (years) }\end{array}$ & $\begin{array}{l}\text { Mutation } \\
\text { origin }\end{array}$ & Region & Nucleotide change & Pain sensation \\
\cline { 3 - 7 } & F & 7 & $\begin{array}{l}\text { Het-F } \\
\text { M }\end{array}$ & $\begin{array}{l}\text { Intron 7 } \\
\text { Intron 2 }\end{array}$ & $\begin{array}{l}\text { c.85I-33T>A } \\
\text { c.287+2dupT }\end{array}$ & + & - \\
pain
\end{tabular}

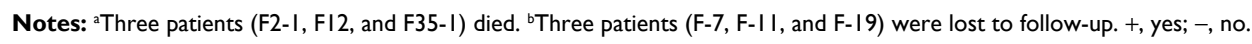

Abbreviations: $F$, female; $M$, male; UN, unknown.

Table 2 Results of thermal testing in five CIPA patients

\begin{tabular}{|l|l|l|l|l|l|l|l|l|l|}
\hline \multirow{2}{*}{ Patients } & \multicolumn{4}{l|}{ Cold $\left(\mathbf{4}^{\circ} \mathbf{C}\right)$} & \multicolumn{2}{l|}{ Warm $\left(\mathbf{4}^{\circ} \mathbf{C}\right)$} & \multicolumn{2}{l|}{ Heat pain $\left(48^{\circ} \mathbf{C}\right)$} \\
\cline { 2 - 10 } & $\begin{array}{l}\text { Dorsum } \\
\text { foot }\end{array}$ & $\begin{array}{l}\text { Dorsum } \\
\text { hand }\end{array}$ & Trigeminal & $\begin{array}{l}\text { Dorsum } \\
\text { foot }\end{array}$ & $\begin{array}{l}\text { Dorsum } \\
\text { hand }\end{array}$ & Trigeminal & $\begin{array}{l}\text { Dorsum } \\
\text { foot }\end{array}$ & $\begin{array}{l}\text { Dorsum } \\
\text { hand }\end{array}$ & $\begin{array}{l}\text { Trigeminal } \\
\text { F-3 }\end{array}$ \\
\hline & + & + & + & - & + & + & + & + & + \\
\hline F-4 & - & - & + & - & - & + & - & - & - \\
\hline F-10 & - & - & - & - & - & + & - & - & - \\
\hline F-28-I, F-28-2 & - & - & - & - & + & + & - & + & + \\
\hline
\end{tabular}

Note: +, yes; -, no.

Abbreviation: CIPA, congenital insensitivity to pain with anhidrosis.

methods mentioned above, mutations in NTRK1 were identified in 36 patients. Finally, a total of 19 mutations (missense, $n=11$; frame-shift, $n=6$; splice, $n=2$ ) were identified (Figure 3).

The mutation sites of 36 patients in this study were analyzed. In all, 23 patients carried the mutation c.851-33T $>$ A (rs80356674) with an incidence of 63.8\%. One pair of sisters (F-16-1, F-16-12) was homozygous of c.851-33T>A, and other patients carrying the mutation were heterozygote. The mutation c.287+2 dupT appeared scattered in six patient pedigrees, and three of the patients had compound heterozygous mutations of c.851-33T >A and c.287+2 dupT (F-15, F-32, and F-34). Based on our previous study, an estimated one out of 968 healthy Chinese people carried the heterozygous variant c.851-33T >A, and none of the 969 people carried the heterozygous variant c. $287+2$ dupT. ${ }^{6}$

\section{Discussion}

This study described the epidemiological status of 41 Han Chinese patients with CIPA and presented preliminary data on the geographical distribution, sex ratio, and mortality rate of this disease. There are currently no reports on CIPA patients in terms of differences in patient sex; however, the results of this study revealed a male to female ratio of 3:1, although the reasons for this difference is not clear. The cause of death was recurrent hyperthermia with a mortality rate of $7.3 \%$, which was lower than the rate of $22 \%$ reported previously in 31 Israeli Bedouin individuals with an average age of 5.2 years; ${ }^{8}$ in that study, the cause of death included infectious diseases, cardiac arrest, or other unknown causes, with the age of death ranging between 40 days and 7 years. ${ }^{18}$

The typical symptoms of CIPA are mostly similar, but there is heterogeneity in details. Generally, whole body areas, including cranial and visceral tissues, lack pain sensation. However, our patients described some pain sensations such as "nose pain" and "stomach pain," which are innervated by trigeminal ganglia neurons and sympathetic visceral sensory afferents, respectively. ${ }^{19}$ In addition, our study included two sisters with the homozygous mutation, c.851-33T $>$ A, who developed pain recovery after 10 years of age. This is a very interesting phenomenon, and similar cases of "recovery" of rare pain have also been reported. A recent study described two brothers with CIPA who were able to perceive pain through periodontal nerves. ${ }^{20}$ A CIPA patient in South Korea was able to perceive painful sensations. ${ }^{21} \mathrm{~A}$ patient of congenital insensitivity to pain with the $S C N 9 A$ mutation 


\begin{tabular}{|l|l|l|l|l|l|l|l|l|}
\hline & No sweat & $\begin{array}{l}\text { Recurrent } \\
\text { fever }\end{array}$ & $\begin{array}{l}\text { Self- } \\
\text { mutilation } \\
\text { ache }\end{array}$ & $\begin{array}{l}\text { Slow } \\
\text { wound } \\
\text { healing }\end{array}$ & $\begin{array}{l}\text { Bone } \\
\text { fractures }\end{array}$ & $\begin{array}{l}\text { Fall } \\
\text { dawn }\end{array}$ & $\begin{array}{l}\text { Severe } \\
\text { infection }\end{array}$ & $\begin{array}{l}\text { Mental } \\
\text { retardation }\end{array}$ \\
\hline+ & + & + & + & - & + & + & - & + \\
\hline- & + & + & + & + & + & - & + & + \\
\hline- & + & + & + & - & & + & + \\
\hline UN & + & + & UN & + & UN & UN & UN & UN \\
\hline+ & + & + & + & + & - & + & - & + \\
\hline
\end{tabular}

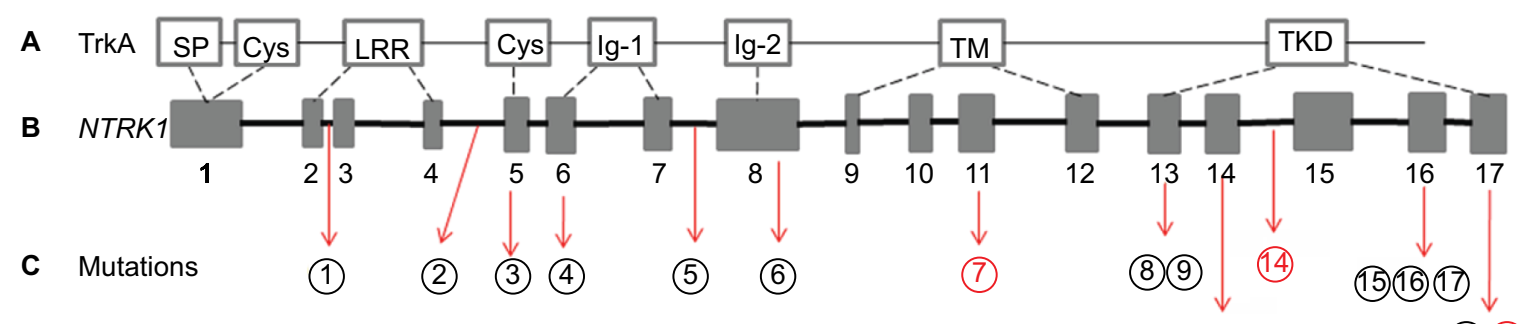
(1) $c .287+2 \mathrm{dupT}$
(2) c.429-374_c.717+485del1403
(3) $c .446 \_47$ insTCTG
(4) $c .632 \mathrm{~T}>\mathrm{A}$
(5) $c .851-33 T>A$
(6) $c .963 \mathrm{delG}$
(7) c.1253_1254delTC
(8) c.1711_1721del
(9) c.1736delT
(10) $c .1775 T>G$

(11) $c .1802 T>G$

(12) c. $1805 \mathrm{G}>\mathrm{A}$

(13) $c .1804 \mathrm{C}>\mathrm{T}$

(14) c. $1806-2 A>G$

(15) $c .2066 \mathrm{C}>\mathrm{T}$

(16) $c .2074 \mathrm{C}>\mathrm{T}$

(17) $c .2197 \mathrm{C}>\mathrm{T}$

(18) c. $2311 \mathrm{G}>\mathrm{A}$

(19) C. $2312 \mathrm{G}>\mathrm{A}$

(18)(19)

Figure 3 Schematic map of NTRKI showing the distribution of mutations identified in Chinese CIPA patients.

Notes: (A) Various structural domains of the TrkA protein. (B) NTRKI consists of 17 exons and spans region of $25 \mathrm{~kb}$. (C) NTRKI mutation spectrum for our patients of CIPA: novel mutations are marked in red, and known mutations are marked in black.

Abbreviations: CIPA, congenital insensitivity to pain with anhidrosis; Cys, cysteine-rich domain; IgC, immunoglobulin-like domain; LRR, leucine-rich domain; SP, signal peptide; TKD, tyrosine kinase; TM, transmembrane domain.

developed neuropathic pain after undergoing a cesarean section. ${ }^{22}$ Additionally, there was a report of paroxysmal abdominal pain in a patient with SCN11A mutations, which resulted in a lack of pain sensation. With this phenomenon, it is difficult to determine whether these patients can perceive pain or if it is due to an acquired behavior to avoid stimuli; however, it can provide different perspectives for our understanding of CIPA patients.

In this disease, temperature perception is also impaired, as consistent errors are made during tests to distinguish between hot and cold substances. ${ }^{1}$ However, the results of our questionnaire and QST show that patients with CIPA experience different levels of temperature sensation. In general, the patients can correctly distinguish between hot and cold stimuli on the head and face, whereas there is loss of temperature perception in the limbs. In addition to thermal pain caused by thermal stimulation in individual patients, almost all of them lacked discrimination between hot and cold pain. NGF not only plays a role in development but also causes sensitizing effects to heat and mechanical stimulation on nociceptors that result in hyperalgesia. NGF-dependent primary afferents are dorsal root ganglia (DRG) neurons or trigeminal ganglia $(\mathrm{V})$ neurons with free nerve endings. ${ }^{2}$

One of the characteristic features of CIPA is multiple accidental injuries to the bone system, such as bone and joint fractures and dislocation of the hip during physical 
development - the most common cause of physical disability. A relatively high incidence of mandibular osteomyelitis was found in patients with CIPA. ${ }^{23}$ In 2014, Haga and Zhang ${ }^{33}$ reported that the most common skeletal complications were fractures (65\%) and Charcot joints (29\%), both involving primarily the lower limbs. Fractures were particularly frequent between ages 1 and 7 years. ${ }^{24}$ The recurring fractures in our patients were mainly limited to the long bones and calcaneus of the lower extremities, as well as hip dislocation or necrosis. Most importantly, they were previously thought to be traumatic fractures caused by painless protection, but most of the patients in this study suffered from unexplained, spontaneous fractures without external forces, which may be related to abnormalities in bone development. Recent studies have found that NGF-TrkA is involved in vascularization and ossification of bone during embryonic development. ${ }^{25,26}$ $\mathrm{X}$-ray and bone mineral density tests revealed severe osteoporosis in the patients. ${ }^{5}$ These symptoms and outcomes may be helpful in studying the effects of TrkA-NGF signaling on bone development and may be helpful in the treatment of the skeletal system in patients with CIPA.

Owing to the lack of pain awareness and protection, wound healing is slow; it has been shown that patients with CIPA are vulnerable to $S$. aureus infection ${ }^{27}$ and that neutrophil chemotactic activity is impaired due to the lack of the NGF/TRKA signaling pathway. ${ }^{28}$

These patients generally have mild to moderate mental retardation. Levy Erez et al $^{34}$ conducted a formal assessment of intelligence and adaptive behavior in 23 children with CIPA and identified an inverse relationship between age and the intelligence quotient in children. In this study, four patients exhibited normal mental development with no apparent delays. However, most of our patients are currently underage, and their mental development needs to be tracked. Franco et $\mathrm{al}^{29}$ showed that TrkA misfolding and aggregation induced by some CIPA mutations disrupt the autophagy homeostasis causing neurodegeneration and generate different levels of cell toxicity.

More than 105 mutations of NTRK1 have been reported to date in CIPA patients globally, and 43 NTRK1 mutations have been reported in Chinese patients so far. ${ }^{3} \mathrm{~A}$ total of 19 mutations were identified in this study, and five of them were novel. In this study, we found that the two most common mutations in the Chinese population were c.851-33T $>\mathrm{A}$ and c. $287+2$ dupT, with mutation rates of $66.7 \%$ and $16.7 \%$, respectively. Miura et al reported in 2000 that the mutation c.851-33C $>\mathrm{T}$ resulted in a splicing abnormality in the seventh intron. ${ }^{11}$ In brief, the mutation c.851-33T $>$ A substitutes
A for a conserved $\mathrm{T}$ ( $\mathrm{U}$ in the transcript) at the position of the branch site and causes aberrant splicing of intron 7 in vitro, resulting in an insertion of a 137-bp segment probably attributable to utilization of an upstream cryptic splice acceptor site and the truncated final TrkA protein. This mutation was also found in patients with CIPA in China, Japan, and Korea. ${ }^{21,30,31}$ Understanding the high incidence of disease-causing mutations in the Chinese population may be instructive for prenatal diagnostic genetic screenings. In addition, there were five patients in this study in whom mutations were not identified using the different sequencing methods. CIPA is currently considered to be a single-gene pathogenic disease caused by mutations in NTRK1. However, Shaikh et $\mathrm{al}^{32}$ conducted a comprehensive functional analysis of seven novel NTRK1 missense mutations and concluded that complete abolition of TRKA kinase activity is not the only pathogenic mechanism underlying CIPA. By corollary, clinical assessment of the pathogenicity of CIPA mutations is more complex than initially predicted and requires a multifaceted approach.

\section{Conclusion}

This study details the clinical phenotypes and mutation characteristics of 41 Chinese CIPA patients to better understand the disease characteristics in Chinese Han patients with CIPA. Furthermore, heterogeneity of clinical features and molecular pathology of CIPA would provide unique opportunities to explore critical roles of the autonomic sympathetic nervous system as well as components of the peripheral sensory nervous system that transmit noxious stimuli in humans.

\section{Acknowledgments}

We thank the patients and their family members for their cooperation. We thank all the medical staff in this study for their intelligence and endeavor.

\section{Author contributions}

All authors contributed to data analysis, drafting or revising the article, gave final approval of the version to be published, and agree to be accountable for all aspects of the work.

\section{Disclosure}

The authors report no conflicts of interest in this work.

\section{References}

1. Swanson AG. Congenital insensitivity to pain with anhydrosis. A unique syndrome in two male siblings. Arch Neurol. 1963;8:299-306.

2. Indo Y. NGF-dependent neurons and neurobiology of emotions and feelings: Lessons from congenital insensitivity to pain with anhidrosis. Neurosci Biobehav Rev. 2018;87:1-16. 
3. Indo Y, Tsuruta M, Hayashida Y, et al. Mutations in the TRKA/NGF receptor gene in patients with congenital insensitivity to pain with anhidrosis. Nat Genet. 1996;13(4):485-488.

4. Indo Y. Nerve growth factor, pain, itch and inflammation: lessons from congenital insensitivity to pain with anhidrosis. Expert Rev Neurother. 2010;10(11):1707-1724

5. Lv F, Xu XJ, Song YW, et al. Recurrent and novel mutations in the NTRK1 gene lead to rare congenital insensitivity to pain with anhidrosis in two Chinese patients. Clin Chim Acta. 2017;468:39-45.

6. Wang QL, Guo S, Duan G, et al. Phenotypes and Genotypes in Five Children with Congenital Insensitivity to Pain with Anhidrosis. Pediatr Neurol. 2016;61:63-69.

7. Haga N, Kubota M, Miwa Z. Epidemiology of hereditary sensory and autonomic neuropathy type IV and V in Japan. Am J Med Genet A. 2013;161(4):871-874.

8. Shatzky S, Moses S, Levy J, et al. Congenital insensitivity to pain with anhidrosis (CIPA) in Israeli-Bedouins: genetic heterogeneity, novel mutations in the TRKA/NGF receptor gene, clinical findings, and results of nerve conduction studies. Am J Med Genet. 2000;92(5):353-360.

9. Mardy S, Miura Y, Endo F, Matsuda I, Indo Y. Congenital insensitivity to pain with anhidrosis (CIPA): effect of TRKA (NTRK1) missense mutations on autophosphorylation of the receptor tyrosine kinase for nerve growth factor. Hum Mol Genet. 2001;10(3):179-188.

10. Indo Y. Molecular basis of congenital insensitivity to pain with anhidrosis (CIPA): mutations and polymorphisms in TRKA (NTRK1) gene encoding the receptor tyrosine kinase for nerve growth factor. Hum Mutat. 2001;18(6):462-471.

11. Miura Y, Mardy S, Awaya Y, et al. Mutation and polymorphism analysis of the TRKA (NTRK1) gene encoding a high-affinity receptor for nerve growth factor in congenital insensitivity to pain with anhidrosis (CIPA) families. Hum Genet. 2000;106(1):116-124.

12. Lin YP, Su YN, Weng WC, Lee WT. Novel neurotrophic tyrosine kinase receptor type 1 gene mutation associated with congenital insensitivity to pain with anhidrosis. J Child Neurol. 2010;25(12):1548-1551.

13. Gao L, Guo H, Ye N, et al. Oral and craniofacial manifestations and two novel missense mutations of the NTRK1 gene identified in the patient with congenital insensitivity to pain with anhidrosis. PLoS One. 2013;8(6):e66863.

14. Wang T, Li H, Xiang J, et al. Identification of a novel nonsense mutation of the neurotrophic tyrosine kinase receptor type 1 gene in two siblings with congenital insensitivity to pain with anhidrosis. $J$ Int Med Res. 2017;45(2):549-555.

15. Geng X, Liu Y, Ren X, et al. Novel NTRK1 mutations in Chinese patients with congenital insensitivity to pain with anhidrosis. Mol Pain. 2018;14:174480691878114.

16. Sagafos D, Kleggetveit IP, Helås T, et al. Single-Fiber Recordings of Nociceptive Fibers in Patients With HSAN Type V With Congenital Insensitivity to Pain. Clin J Pain. 2016;32(7):636-642.

17. Wang Q, Guo S, Duan G, et al. Novel and novel de novo mutations in NTRK1 associated with congenital insensitivity to pain with anhidrosis: a case report. Medicine (Baltimore). 2015;94(19):e871.

18. Loewenthal N, Levy J, Schreiber R, et al. Nerve growth factor-tyrosine kinase A pathway is involved in thermoregulation and adaptation to stress: studies on patients with hereditary sensory and autonomic neuropathy type IV. Pediatr Res. 2005;57(4):587-590.
19. Indo Y. Nerve growth factor and the physiology of pain: lessons from congenital insensitivity to pain with anhidrosis. Clin Genet. 2012;82(4):341-350.

20. Guven Y, Altunoglu U, Aktoren O, et al. Twins with hereditary sensory and autonomic neuropathy type IV with preserved periodontal sensation. Eur J Med Genet. 2014;57(5):240-246.

21. Jung CL, Ki CS, Kim BJ, et al. Atypical hereditary sensory and autonomic neuropathy type IV with neither mental retardation nor pain insensitivity. J Child Neurol. 2013;28(12):1668-1672.

22. Wheeler DW, Lee MC, Harrison EK, Menon DK, Woods CG. Case Report: Neuropathic pain in a patient with congenital insensitivity to pain. F1000Res. 2014;3:135.

23. Machtei A, Levy J, Friger M, Bodner L. Osteomyelitis of the mandible in a group of 33 pediatric patients with congenital insensitivity to pain with anhidrosis. Int J Pediatr Otorhinolaryngol. 2011;75(4): 523-526.

24. Phatarakijnirund V, Mumm S, Mcalister WH, et al. Congenital insensitivity to pain: Fracturing without apparent skeletal pathobiology caused by an autosomal dominant, second mutation in SCN11A encoding voltage-gated sodium channel 1.9. Bone. 2016;84:289-298.

25. Tomlinson RE, Li Z, Zhang Q, et al. NGF-TrkA Signaling by Sensory Nerves Coordinates the Vascularization and Ossification of Developing Endochondral Bone. Cell Rep. 2016;16(10):2723-2735.

26. Tomlinson RE, Li Z, Li Z, et al. NGF-TrkA signaling in sensory nerves is required for skeletal adaptation to mechanical loads in mice. Proc Natl Acad Sci U S A. 2017;114(18):E3632-E3641.

27. Hepburn L, Prajsnar TK, Klapholz C, et al. Innate immunity. A Spaetzle-like role for nerve growth factor $\beta$ in vertebrate immunity to Staphylococcus aureus. Science. 2014;346(6209):641-646.

28. Beigelman A, Levy J, Hadad N, et al. Abnormal neutrophil chemotactic activity in children with congenital insensitivity to pain with anhidrosis (CIPA): the role of nerve growth factor. Clin Immunol. 2009;130(3): 365-372.

29. Franco ML, Melero C, Sarasola E, et al. Mutations in TrkA Causing Congenital Insensitivity to Pain with Anhidrosis (CIPA) Induce Misfolding, Aggregation, and Mutation-dependent Neurodegeneration by Dysfunction of the Autophagic Flux. J Biol Chem. 2016;291(41):21363-21374.

30. Lee ST, Lee J, Lee M, Kim JW, Ki CS. Clinical and genetic analysis of Korean patients with congenital insensitivity to pain with anhidrosis. Muscle Nerve. 2009;40(5):855-859.

31. Nam TS, Li W, Yoon S, et al. Novel NTRK1 mutations associated with congenital insensitivity to pain with anhidrosis verified by functional studies. J Peripher Nerv Syst. 2017;22(2):92-99.

32. Shaikh SS, Chen YC, Halsall SA, et al. A Comprehensive Functional Analysis of NTRK1 Missense Mutations Causing Hereditary Sensory and Autonomic Neuropathy Type IV (HSAN IV). Hum Mutat. 2017;38(1):55-63.

33. Zhang Y, Haga N. Skeletal complications in congenital insensitivity to pain with anhidrosis: a case series of 14 patients and review of articles published in Japanese. J Orthop Sci. 2014;19(5):827-831.

34. Levy Erez D, Levy J, Friger M, Aharoni-Mayer Y, Cohen-Iluz M, Goldstein E.Assessment of cognitive and adaptive behaviour among individuals with congenital insensitivity to pain and anhidrosis. Dev Med Child Neurol. 2010;52(6):559-62. 


\section{Supplementary material}

$\mathrm{F}-1$

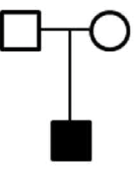

c.287+2dupT

c. $1775 T>G$

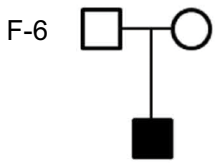

c. $851-33 \mathrm{~T}>\mathrm{A}$

$\mathrm{F}-11$

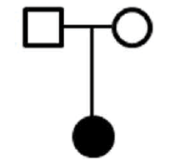

c. $851-33 \mathrm{~T}>\mathrm{A}$

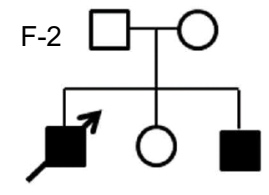

c.429-374_c. $717+485$ del1403

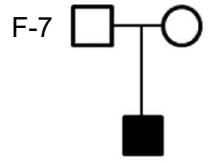

c. $287+2$ dupT

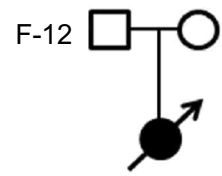

c. $851-33 \mathrm{~T}>\mathrm{A}$
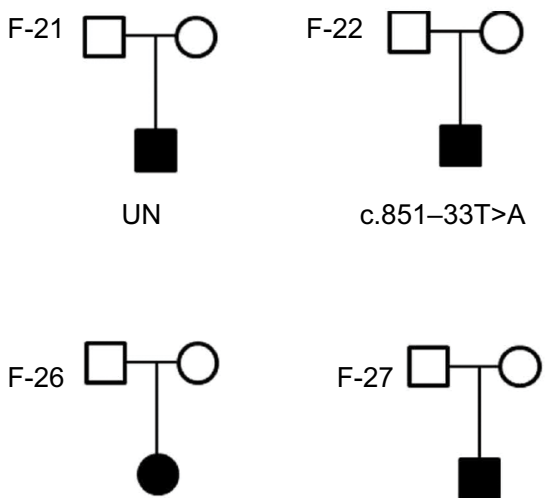

c.851-33T>A c.1710_1720del

F-3

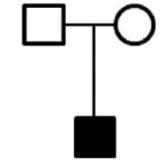

c. $287+2$ ins $T$

c.446_447insTCTG

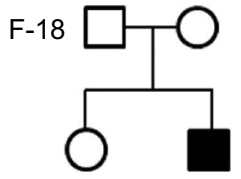

UN

c.851-33T >A

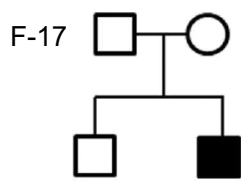

UN

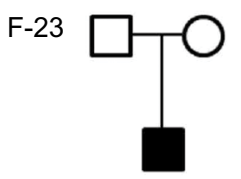

c.851-33T >A

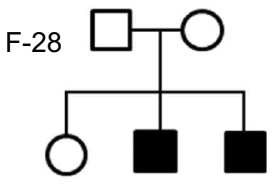

c. $632 \mathrm{~T}>\mathrm{A}$

c.1253_1254delTC

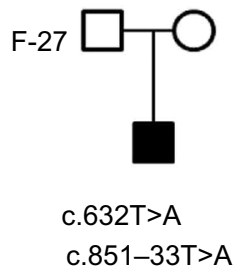

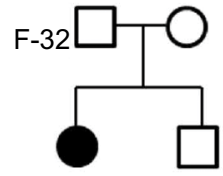

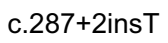

c.851-33T>A
F-3

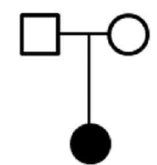

c.446_447ins TCG

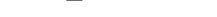

F-8

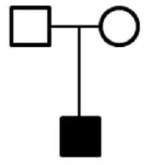

c.851-33T>A

F-13

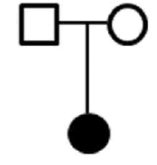

c.1646delT

F-4

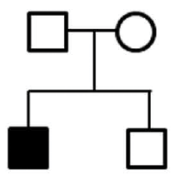

c. $2074 \mathrm{C}>\mathrm{T}$

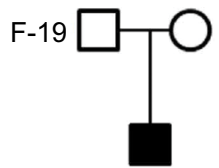

F-24

F-29

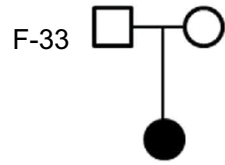

c.446_447insTCTG c.815-33T >A
F-5

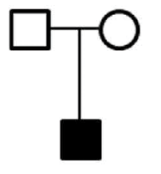

c. $2074 \mathrm{C}>\mathrm{T}$ c. $2311 \mathrm{C}>\mathrm{T}$
F-9

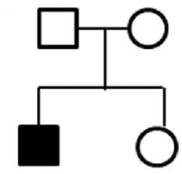

c. 851-33T>A c. $2197 \mathrm{G}>\mathrm{A}$

F-14

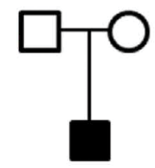

c.851-33T>A

c. $1806-2 A>G$ c. $2312 \mathrm{G}>\mathrm{A}$

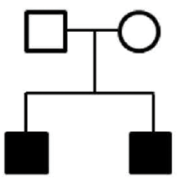

c.851-33T>A c.963delG

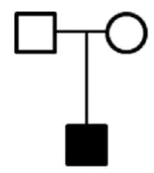

c.851-33T $>A$ c. $1805 \mathrm{G}>\mathrm{A}$

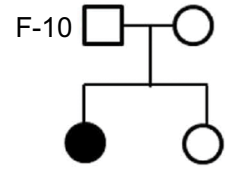

UN

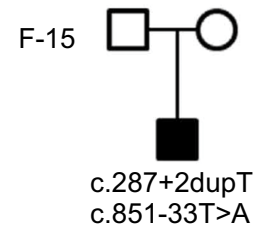

F-20

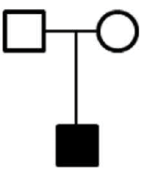

UN

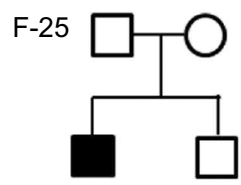

c. $1802 \mathrm{~T}>\mathrm{G}$

c. $1804 \mathrm{C}>\mathrm{T}$

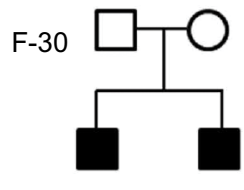

c.851-33T >A c. $2066 \mathrm{C}>\mathrm{T}$

F-34

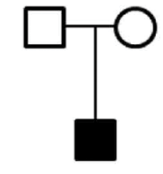

c. $287+2$ ins $T$ c. 851-33T $>$ A

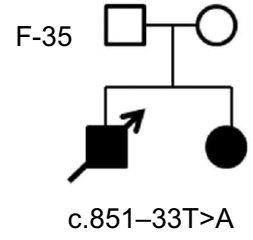

Figure SI Pedigrees of the families with NTRKI mutations of CIPA patients in 35 families. Notes: The black oblique arrow indicates that the patient has died. 
The Journal of Pain Research is an international, peer reviewed, open access, online journal that welcomes laboratory and clinical findings in the fields of pain research and the prevention and management of pain. Original research, reviews, symposium reports, hypothesis formation and commentaries are all considered for publication
The manuscript management system is completely online and includes a very quick and fair peer-review system, which is all easy to use. Visit http://www.dovepress.com/testimonials.php to read real quotes from published authors.

Submit your manuscript here: https://www.dovepress.com/journal-of-pain-research-journal 